\title{
Autosomal Dominant Polycystic Kidney Disease
}

National Cancer Institute

\section{Source}

National Cancer Institute. Autosomal Dominant Polycystic Kidney Disease. NCI

Thesaurus. Code C84578.

Polycystic kidney disease inherited in an autosomal dominant pattern. Symptoms usually appear at middle age and include abdominal pain, hematuria and high blood pressure.

Patients may develop brain aneurysms and liver cysts. 